\title{
Nano and Microtechnology Emerging Impact on Pharmacology and Medical Advances: Prospects and Challenges-Mini Review
}

\author{
Obafunso A Ajayi ${ }^{1 *}$ and Adesuyi A Leslie Ajayi ${ }^{2}$ \\ ${ }^{1}$ Columbia Technology Ventures, Columbia University, USA \\ ${ }^{2}$ Hypertension and Clinical Pharmacology Division, Baylor College of Medicine, USA
}

Submission: April 26, 2017; Published: May 04, 2017

*Corresponding author: Adesuyi A Leslie Ajayi, MD, PhD, Division of Hypertension and Clinical pharmacology, Baylor College of Medicine, Houston, TX 77060, USA, Tel: 83240 60772; Email: demi.ajayi@columbia.edu

\begin{abstract}
We conducted a focused review of the emerging impact of micro and nanoscale engineered products (nano structures or nano vehicles) on the future efficacy and safety of old and newer medicines or biologics. The combination of medicines and biologics with nano vehicles constitute nano medicines. The size of these nano medicines in use range from $10-1000 \mathrm{~nm}$, which crosses the boundaries of nano to micro technology. The arena of greatest advance was in the preclinical and clinical development of novel engineered drug delivery systems through the use adjunctive nano vehicles, which permitted greater site of action drug delivery and increased concentrations, targeted site delivery by passive and active targeting, delivery into hard to reach areas (including brain, eyes, intracellular sites), and cell nucleus. These developments hold the promise to reduce systemic adverse effects of toxic drugs, such as seen with cancer chemotherapeutic drugs, reduce systemic adverse drug reactions, and overcome multidrug drug (MDR) resistance. There are issues of local toxicity arising from prolonged half life and increased residence time of PEGlylated liposomes bearing anticancer agents. Nano medicines that are site targeted also have a role in personalized medicine. Taken together, the l engineered nano vehicles have a promising impact, to translate to improved therapeutic outcomes in a wide variety of clinical situations, whilst also achieving some unmet therapeutic goals, but the issue of safety of the biodegradable products, and the cost and cost-effectiveness remain unanswered.
\end{abstract}

Keywords: Nano medicines; Nano vehicles; Cancers; Brain; Eye; Nano toxicity

Abbreviations: BBB: blood brain barrier; PEG: Polyethylene Glycol; ALL: Acute lymphocytic Leukemia; AMD: Age related -Macular Degeneration; SiRNA: Small interfering RNA; f-CNT: Functionalized Carbon Nano tubes; SWCNT: Single Walled CNT; MWCNT: Multi-walled CNT; VEGF: Vascular Endothelial Growth Factor; CNTMTT: CNT mediated Thermal Therapy; EPR: Enhanced and Permeation Retention; MDR: Multi-Drug Resistance

\section{Introduction}

Nanostructures are engineered materials with one of the dimensions ranging from $1-100 \mathrm{~nm}$, whilst microstructures are devices or products ranging from 1 micrometer and above. The physical, chemical, optical properties of these materials at this size tend to differ from the bulk substances from which they were made [1]. One major advantage of nano particles or nano vehicles is the possession of a high surface area to weight ratio, this permits their greater reactivity with molecules and ligands than larger ones in standard drug formulations, leading to putatively greater efficacy $[1,2]$. The various types of smart nano vehicles with tunable physicochemical properties range from drug-polymer conjugates, micro particles up to 100 micrometers in size, protein filled nano particles, micelle- polymers and liposomes and other temperature or body $\mathrm{PH}$ sensitive structures [1-5]. The current reviews discusses the nano engineered materials and their properties, focuses on how the nano vehicles transport drugs, genes or biologicals to 'sanctuary sites" which are hard to reach for conventional drug delivery systems, owing to barriers to drug penetration. These include areas, such as the brain and brain or brain neoplasms, due to 
the blood brain barrier (BBB), ocular pharmacology, because of the blood /ocular and blood / retinal barriers, inflammation or infections. Nanotechnology based imaging is beyond the scope of this review.

\section{Nature of the Nano-micro engineered products}

The nano medicines consist of the conventional drug, biological products such as genes, small interfering or silencing RNA (siRNA), antibodies, in combination with the nano-micro engineered transporting vehicle or Nano vehicle [3,4]. Most of the nano medicines in use or development range in size from $10 \mathrm{~nm}$ to $1000 \mathrm{~nm}$, thus encompassing both nanotechnology and micro technology. The nano vehicle thus serves as the new but engineered drug excipient but designed to carry a much smaller payload of drugs or biologicals, targeted to achieve greatest concentration at a predetermined site of the body or tissue. The nano medicine (drug/biological + nano vehicle) can be classified broadly as

\section{i. Inorganic or}

ii. Organic.

Inorganic nano medicines have metals and can be magnetically tuned and trafficked or guided. Examples include gold used for thermal ablation of tumors, iron oxide used for tumor abalation, imaging, and in the treatment of refractory iron deficiency and in patients with anemia of chronic renal failure $[2,3,5]$. Silver and Silica have been employed as nano porous particles in the treatment of bacterial infections. Clofazimine and other agents have been encapsulated in in silica to treat antibiotic resistant Mycobactrium Tuberculosis [6]. Organic nano vehicle transported medicines are designed to cross cellular or tissue barriers which otherwise are pharmacokinetically difficult to reach.

Perhaps the most important and one of the earliest are the liposomes. Liposomes [3,4] are circular or vesicular ranging in size from $20 \mathrm{~nm}-100 \mathrm{~nm}$ and have a phospholipid bilayer structure, enclosing aqueous or hydrophilic content. The drug or biologic is enclosed in the inner aqueous region. The liposomes, owing to their size and charge, increase bioavailability to the targeted site of action. They release their drug content in a slow release manner, and thus increase the residence time of the drug. The site of action concentration $\left(\mathrm{C}_{\mathrm{S}}\right)$ of the drug is thereby elevated over a prolonged time [3]. Liposomes can be surface engineered to have receptor specific ligands which can increase penetrance. Coating with Polyethylene Glycol (PEG) achieves PEGY-lated liposomes, which have conferred greater bioavailability, increased drug residence time and greater pharmacodynamics. PEGylation of liposomes also confers biocompatibility on the nanomedicine, and allows it to escape from phagocytosis by cells of the reticulo-endothelial system. This ability to evade immune response confers a stealth ability, which non-PEGylated liposomes lack [3,4]. Examples of liposomes as nano vehicles include cancer chemotherapeutic drugs including Doxil which contains doxorubicin in a PEGylated liposome.

Many cancer chemotherapeutic agents such as Daunorubicin for primary HIV Kaposi Sarcoma, Endotag-1, a liposomal Paclitaxel used to treat triple negative breast cancers and pancreatic cancers, Marqibo, PEGylated Vincristine Philadelphia chromosome negative, Acute lymphocytic Leukemia (ALL) and some brain tumors. In addition to these, irinotecan for metastatic pancreatic cancer and brain tumors are FDA approved liposome delivered nano medicines. Others in clinical use are antifungal drugs, Ambisome, liposomal amphotericin B, Diprivan (anesthetic propfol in liposomes), Visudyne, a liposomal verteporfin, used in the treatment of Age related -macular degeneration (AMD). Small interfering RNA (SiRNA) are also administered to reach the nuclear targets and DNA via liposome as nano vehicles. Protein filled nano medicines are usually small molecules attached as adjuncts to albumin to form drug -albumin complexes. Unlike, PEGylated liposomes, these can excite an immune response in the host, by phagocytosis of the nano medicines by macrophages.

Abraxane, which combines anticancer drug paclitaxel to albumin, is an example. It is FDA approved for treating inoperable non small pulmonary cancer, metastatic breast and pancreatic cancer. Dipepetide nano particles have also being engineered to be to be fluorescent for target cancer cell imaging and real time monitoring of the drug release [7]. The dual ability and utility both to diagnose and treat by the same nano medicine, is an example "theragnostic" nano medicine. Polymeric micelle nano medicines are drug -polymer conjugates, which are biodegradable and often used to ferry drugs. They are have increased attachment to site of action and controlled release of the constituent drugs. They entrap drugs within micelles and release at the targeted point of action. Other agents' nano vehicles include, dendrimers, which are branched polymers and thus attach. Many of the organic nano medicines are administered via the intravenous route to achieve their engineered functions. Some are topically administered for transdermal drug delivery and as eye drops, such as Restasis (containing cyclosporine A) for chronic dry eye.

Functionalized Carbon Nano tubes (f-CNT) [8] are onedimensional (1D) nano vehicles, which can be single walled CNT (SWCNT) or Multi-walled CNT (MWCNT). These CNT have made made biocompatible for in vivo use, and functionalized by coating with proteins. PEGylated -SWCNT are conjugated with ligands against integrins -alpha vb-3 receptors expressed on tumor blood vessels. Circulating f-CNT are selectively taken up by macrophage/monocyte phagocyte for enhanced spread and especial delivery to tumors. F-CNTs are of great promise as nano vehicles for brain delivery of drugs and genes [8]. They are taken up across more easily by cells of the BBB, by micro pinocytosis, receptor -mediated transcytosis. The f-CNT are very promising in vivo nano vehicles in mouse models against brain diseases such as Alzheimer's dementia - with SWCNT bearing 
acetylcholine, and for glioblastoma and stoke. Functionalized -SWCNT has also been used to deliver caspase-3 siRNA, for gene silencing to induce apoptosis in rat stroke models $[3,8]$.

\section{Crossing barriers to sanctuary sites of the brain and the eye}

A major goal and emerging advantage of nano medicines is to ensure drug delivery to the hard to reach or sanctuary sites, which are protected from drug or xenobiotic entry.

\section{Ocular drug delivery: pharmacokinetics pharmacodynamics}

and

Eye diseases such as glaucoma, ocular tumors, macular degeneration and diabetic proliferative retinopathy are leading causes of blindness, globally. Current drug treatment of eye diseases entails use of emulsions, topical eye drops which may reach the anterior eye segment of conjunctiva to the iris, but have only $5 \%$ bioavailability for posterior segment eyes diseases, and $2 \%$ bioavailabilty at the retina [9-11]. Part of the reason for this poor penetration and bioavailability, include tears and lachrymal secretions which reduce drug penetration, poor vitreous permeability and blood/vitreal barriers blood retinal barriers. Intravitreal drug injections for posterior segment eye disease is effective but of short lived efficacy and cumbersome, but other routes are now preferred. Ocular drug delivery has the potential of being improved by nano medicines. Dexamethasone has US Food and Drug Administration approval as dexamethasone implants to treat macular edema; secondary to retinal vein thrombosis and posterior segment non infectious uveitis.

Dexamethasone half life in the vitreous humor is thereby increased by these dexamethasone liposomes and dendrimers show great promise [9,10]. Restasis is a FDA approved ophthalmic emulsion for chronic dry eye containing cyclosporine A. Liposomes in development for clinical use are being injected to juxtascleral space of the eye, subconjuctiva and subtonal spaces to reduce eye inflammation. Anti-VEGF (Vascular Endothelial Growth Factor) as micropsheres or liposomes have been approved clinically for diabetic neo vascularization treatment. Visudyne (photo-activated liposomal verteporfin has FDA approval for treating choroidal neo vascularization following Agrelated macular degeneration and ocular histoplasmosis [11]. In the anterior eye chamber, dexamethasone, prednisolone, and timolol in nano vehicles cause slow release and used to treat postoperative eye inflammation and chronic glaucoma $[3,4,9,10]$.

\section{Brain drug delivery in vivo models [12-14]}

The blood brain barrier (BBB) is a neuro protective impediment to most drugs or xenobiotics gaining entry into the cerebrospinal axis and fluid. The barrier is entails tight capillary endothelial junctions, and astrocytes as mechanical impediments, as well efflux proteins such as P-1 glycoprotein which pump out most drugs from the brain cerebrospinal fluid. This physiological mechanism reduces central nervous penetration of cancer chemotherapeutic agents, thus limiting effective treatment of brain and spinal neoplasms. Use of functionalized nano vehicles, with surface modifications with specific ligands attached, enables crossing the blood brain barrier and drug release along concentration gradient. Solid lipid nano particles by coacervation and loaded with APO-E -emethotrexate prodrug was shown to be effective against brain gliomas, in a recent study [14]. A combination of liposomes loaded with anticancer drugs, and functionalized CNT has been used in preclinical models in mice for systemic or stereotaxic intracranial administration of SWCNT loaded with immune-adjuvants or oligo nucleotides in mouse glioblastoma models [8]. Glioblastoma multiforme is the brain cancer with the worst prognosis, and silver lining now exists in its treatment with modalities and CNT mediated Thermal Therapy (CNTMTT) [8,12-14].

\section{Active and passive targeting of tumors}

Tumors of the brain and elsewhere have neoplastic vascularization with permeable endothelial lining. Tumor vasculatures also have poor lymphatic drainage $[3,4,12]$. This confers the attribute that is exploited to create Enhanced and Permeation Retention (EPR) effect of anticancer nano medicines in tumors. The small $(20-100 \mathrm{~nm})$ nano vehicle agents are able to penetrate cancer vasculature but not the normal vasculature, which are less permeable. The anticancer drug are then released and retained at the tumoral circulation, by eluting to the perivascular sites of the tumor to exert their cytotoxic action in a site specific manner. The preferential accumulation of nano medicine in tumor vasculature by EPR effect is passive targeting of tumors. Active targeting of tumors entails the deliberate coating of the surfaces of nano vehicles, such as liposomes or f-CNT or dendrimers, with ligands which are specific for receptors that are over-expressed in the tumors, but not in normal cells. These permit tumor -specific interaction of the nano medicines for cytotoxic action sparing the normal cells. Such "smart" nanoengineerd ligands include transferrin and folic acid moieties which are then bound to the cancer cells $[3,4,12]$. These novel nano medicines are thus able to overcome and penetrate tumor cells in adequate concentrations, as to overcome Tumor MultiDrug Resistance (MDR), which causes efflux from tumor/cancer cells owing to enhanced p1-glycoprotein over expression in such tumor cells [4].

\section{Challenges still facing nano medicines}

Despite the exciting prospects of nano medicines, many challenges remain. Although some have gained clinical use, many are yet to be translated from preclinical pharmacology to clinical trials or human use [3-8]. The issue of the safety of the biodegradable nano vehicles remain, and few comparisons of the pharmacodynamics and efficacy of different nano vehicles bearing the same drug for the same disease model exists. This hiatus in knowledge needs to be rectified. There exists also, concerns about the safety and toxicity of the drug payload. There is evidence of prolonged residence time by an order of 
magnitude, especially of anticancer medications causing local and systemic adverse effects [3-5]. Further, these engineered nano medicines are expensive and cost and cost-benefit, cost -effectiveness analyses are required.

\section{Conclusion}

Nanotechnology is slowly but surely revolutionizing pharmacology and clinical practice, but the enterprise is still in infancy. There are still many areas and drugs with preclinical pharmacology studies, but which are yet to attain clinical use and approval. Concerted basic and translational research across many disciplines are required to achieve the full potential, and to assess realistically the safety, risk, cost effectiveness and cost -benefit of emerging nano medicines. Nano toxicity to individuals and to the environment are the issues that need resolution before these smart nano medicines achieve full acceptance in pharmacology and clinical practice.

\section{References}

1. Farokzad OC, Langer R (2009) Impact of nanotechnology on drug delivery. ACS Nano 3(1): 16-20.

2. Ozdemir V, William-Jones B, Glatt SJ, Tsung MT, Lohr JB, et al. (2006) Shifting emphasis from pharmacogenomics to theragnostics. Nature Biol 24(8): 942-946.

3. Anselmo AC, Mitragotri S (2016) Nanoparticles in the clinic. Bioengineering and Translational Medicine 1(1): 10-29.

4. Pillai G (2014) Nanomedicines for Cancer Therapy: An update of FDA approved and those under various stages of development. SOJ Pharm Pharm Sci 1(2): 13.
5. Samanta S, Bagnanowicz DR, Lu HH, Koberstein JT (2016) Polyacetals: water soluble, Ph degradable polymers with extraordinary temperature response. Macromolecules 49(5): 1858-1864.

6. Valetti S, Xia X, Costa-Gouveia J, Brodin P, Bernet Camard MF, et al. (2017) Clofamizine encapsulation in nanoporous silica particles for oral treatment of antibiotic resistant mycobacterium tuberculosis infections. Nanomedicine (Lond) 12: 831-844.

7. Fan Z, Sun L, Huang Y, Wang Y, Zhang M (2016) Bionspired fluorescent dipeptide nanoparticles for target cancer cell imaging and real time monitoring and drug release. Nature Nanotechnology 11: 388-395.

8. Wang J T-W, Al-Jamar KT (2015) Functionalized Carbon nanotubes: revolution in brain delivery. Nanomedicine (Lond) 10(17): 2639-2642.

9. Villanueva JR, Villanueva LR, Navarro MG (2017) Pharmaceutical technology can turn a traditional drug, dexamethasone into a first line ocular medicine: A global perspective and future trends. Int J Pharmaceutics 516(1-2): 342-351.

10. Fangueiro JF, Velga F, Silva AM, Souto EB (2016) Ocular drug delivery -New strategies for targeting Anterior and Posterior segments of the eye. Current Pharm Design 22(19): 1135-1146.

11. Shen HH, Chan EC, Lee JH, Bee YS, Lin TW, et al. (2015) Nanocarriers for treatment of ocular vascularization in the back of the eye: new vehicles for ophthalmic drug delivery. Nanomedicines (Lond) 10 (13): 2093-2107.

12. Tzeng SY, Green JJ (2013) Therapeutic nanomedicine for brain cancer. Ther Deliv 4(6): 1.

13. Meng J, Agrahari V, Youm I (2017) Advances in targeted drug delivery approaches for central nervous tumors : The inspiration of nanobiotechnology. J Neuroimmune Pharmacol 12(1): 84-89.

14. Battaglia L, Muntoni E, Chirio D (2017) Solid lipid particles by coacervation loaded with methotrexate prodrug : preliminary study of glioma treatment. Nanomedicine (Lond) 12(6): 639-656.

\begin{tabular}{l} 
Your next submission with Juniper Publishers \\
will reach you the below assets \\
- Quality Editorial service \\
- Swift Peer Review \\
- Reprints availability \\
- E-prints Service \\
- Manuscript Podcast for convenient understanding \\
- Global attainment for your research \\
- Manuscript accessibility in different formats \\
( Pdf, E-pub, Full Text, Audio) \\
- Unceasing customer service \\
Track the below URL for one-step submission \\
https://juniperpublishers.com/online-submission.php \\
\hline
\end{tabular}

